\title{
Global Wind Speed Retrieval From SAR
}

\author{
Jochen Horstmann, Helmut Schiller, Johannes Schulz-Stellenfleth, and Susanne Lehner, Member, IEEE
}

\begin{abstract}
The global availability of synthetic aperture radar (SAR) wave mode data from the European Remote Sensing (ERS) satellites ERS-1 and ERS-2, as well as ENVISAT, allows for the investigation of the wind field over the ocean on a global and continuous basis. For this purpose, 27 days of ERS-2 SAR wave mode data were processed, representing a total of 34310 imagettes of size $10 \mathrm{~km} \times 5 \mathrm{~km}$, available every $200 \mathrm{~km}$ along the satellite track. In this paper, two methods for retrieving wind speeds from SAR imagettes are presented and validated, showing the applicability of ENVISAT alike SAR wave mode data for global ocean wind retrieval. The first method is based on the well-tested empirical C-band scatterometer (SCAT) models, which describe the dependency of the normalized radar cross section (NRCS) on wind speed and direction. To apply C-band models to SAR data, the NRCS needs to be accurately calibrated. This is performed by a new efficient method utilizing a subset of colocated measurements from ERS-2 SCAT and model winds from the European Centre for Medium-Range Weather Forecast (ECMWF). SAR wind speeds are computed from the calibrated imagettes and compared to the entire set of colocated ERS-2 SCAT and ECMWF model data. Comparison to ERS-2 SCAT winds result in a correlation of 0.95 with a bias of $-0.01 \mathrm{~ms}^{-1}$ and an rms error of $1.0 \mathrm{~ms}^{-1}$. The second approach is based on neural networks (NNs), which allow the retrieval of wind speeds from uncalibrated SAR imagettes. NNs are trained using the mean intensity of ERS-2 SAR imagettes and colocated wind data from the ERS-2 SCAT and ECMWF model data. Validation of the NN-retrieved SAR wind speeds to ERS-2 SCAT and ECMWF model wind data result in a correlation of 0.96 with a bias of $-0.04 \mathrm{~ms}^{-1}$ and an rms error of $0.93 \mathrm{~ms}^{-1}$.
\end{abstract}

Index Terms-Calibration, neural network, scatterometer, synthetic aperture radar (SAR), wind speed.

\section{INTRODUCTION}

$\mathbf{S}$ INCE THE LAUNCH of the European Remote Sensing (ERS) satellites ERS-1 and ERS-2 in 1991 and 1995, synthetic aperture radar (SAR) imagettes have been acquired over the oceans on a continuous basis. Full-swath SAR images of $100 \mathrm{~km} \times 100 \mathrm{~km}$ size are taken where receiving stations are in line of sight (image mode), whereas $10 \mathrm{~km} \times 5 \mathrm{~km}$ SAR wave mode data are acquired every $200 \mathrm{~km}$ along the satellite track and, therefore, yield global and continuous observations of the ocean surface during the last decade. Since March 2002, the ERS-2 SAR era has been continued by the Advanced SAR (ASAR) aboard the ENVISAT satellite, acquiring wave

Manuscript received August 12, 2002; revised February 10, 2003. This work was supported by the European Union and by the German Bundesministerium für Bildung und Forschung in the framework of the ENVOC project and by the European Union in the MaxWave project.

J. Horstmann and H. Schiller are with the Institute for Coastal Research, GKSS Research Center, Geesthacht 21502, Germany.

J. Schulz-Stellenfleth and S. Lehner are with the German Aerospace Center (DLR), DFD-AP, Oberpfaffenhofen 82230, Germany.

Digital Object Identifier 10.1109/TGRS.2003.814658 mode data every $100 \mathrm{~km}$ along its satellite track. Due to their all-weather capability and high resolution, SAR systems have become a valuable tool for measuring marine parameters such as wind fields [1], [2], ocean wave spectra [3], [4], and sea ice variables [5].

Many studies on wind retrieval from calibrated spaceborne SAR images have been performed [1], [2], [6]-[9]. All of these rely on well-calibrated SAR images of the satellites ERS-1, ERS-2, and the Canadian satellite RADARSAT-1 and are limited to regional applications. In all of these studies, wind speed is retrieved from the calibrated normalized radar cross section (NRCS) of the ocean surface using the empirical C-band models, which were originally developed for the ERS-1 scatterometer (SCAT) [10], [11].

A new approach to retrieve wind speeds from SAR data is based on the application of neural networks (NNs) [12]. NNs have been used in several applications in remote sensing, e.g., derivation of water properties from imaging spectrometers [13]. Furthermore, Thiria et al. [14] and Richaume et al. [15] applied NNs to wind retrieval from spaceborne SCAT data and ERS-1 SCAT data.

In this study, a unique set of single-look complex ERS-2 SAR wave mode imagettes, which are not available as a standard product from the European Space Agency (ESA), is used for wind speed retrieval on a global and continuous basis. In total 34310 ENVISAT-like SAR imagettes were processed at the German Aerospace Centre representing 27 days of data. Previous studies on the use of ERS wave mode data for wind speed measurements were already published by Kerbaol et al. [16] and Lehner et al. [17]. However, a calibration and subsequent determination of wind speeds has not been previously undertaken. In this paper, two methods for retrieving wind speeds from SAR imagettes are applied and validated, showing their applicability for continuous global ocean wind retrieval.

Different spaceborne sensors (e.g., the altimeter, radiometer, SCAT, and SAR) exist, which provide information on the nearocean surface wind fields on a regional and global scale. All these sensors rely on the fact that the small-scale sea surface roughness is closely related to the local wind field. However, it is well known that this connection is not simple, as the surface roughness is also affected by features such as rain, fronts, surface slicks, or sea ice, for example. SAR scenes give a direct indication of these artifacts, while the other sensors often do not detect these features and misinterpret them as wind. It is also well known that surface gravity waves that have been generated by wind fields in distant areas can have an impact on the wind retrieval [18]. The accuracy of wind measurement techniques has now reached a level where these effects have to be addressed in order to achieve further improvements. In this context, SAR imagette data have great potential, as they provide information 
on both the small-scale surface roughness and the longer ocean surface waves. The calibration method presented in this paper also has an important technical application, i.e., the monitoring of the radiometric performance of a SAR on a global and continuous basis.

This paper is organized as follows. In Section II, the available data are introduced. Section III shows the dependency of SAR imagettes mean intensity on wind speed and direction by comparison to ECMWF model and ERS-2 SCAT data. In Section IV, wind speeds are retrieved from SAR imagettes using the C-band SCAT model CMOD4. For this purpose the amplitudes of the SAR imagettes are calibrated to NRCS using a new efficient method. The resulting wind speeds are compared to colocated ECMWF model and ERS-2 SCAT data. In Section V, wind speeds are derived from SAR imagettes using NNs. The NN-retrieved wind speeds are compared to colocated ECMWF model and ERS-2 SCAT data. In Section VI, the main differences between wind speeds retrieved from SAR imagettes, ECMWF model results and ERS-2 SCAT data are identified and differences are discussed.

\section{INVESTIGATED DATASETS}

The ERS-2 satellite operates in a near-circular polar and sunsynchronous orbit at a mean altitude of $785 \mathrm{~km}$. The satellite has a repeat cycle of 35 days with an orbital period of $\sim 100 \mathrm{~min}$, resulting in 14.3 orbits per day. The platform is equipped with a SAR and a SCAT combined in the active microwave instrument (AMI), which operates with a frequency of $5.3 \mathrm{GHz}$ (C-band) and can transmit and receive with linear vertical polarization. The AMI can be operated in an interleaved mode consisting of SAR wave mode and SCAT measurements. In SAR wave mode, $10 \mathrm{~km} \times 5 \mathrm{~km}$ imagettes are acquired at a nominal incidence angle of $23^{\circ}$ with a spatial resolution of $\sim 30 \mathrm{~m}$ every $200 \mathrm{~km}$ along the orbit. These imagettes are processed at ESA into image power spectra (UWA), which are used for global ocean wave retrieval [3]. However, for wind retrieval, SAR wave mode data have to be processed to single-look complex imagettes, which to date are not available as a standard product for ERS, but will be available from the ASAR aboard ENVISAT. To prepare and develop algorithms for the ENVISAT ASAR, the German Aerospace Center processed 27 days of ERS-2 SAR wave mode raw data to SAR imagettes between August 21, 1996 and June 2, 1997 [17] using their research processor BSAR [19]. Due to the lack of measurements needed for radiometric calibration, SAR imagettes are not radiometrically calibrated for NRCS. In Fig. 1, the distribution of the entire set of processed SAR imagettes over the globe is shown.

In contrast to the SAR, the ERS-2 SCAT measures the backscatter from the ocean surface with three antennae looking $45^{\circ}$ forward, sideways, and $45^{\circ}$ backward with respect to the satellite flight direction. These beams continuously illuminate a 500 -km-wide swath with a resolution of $45 \mathrm{~km}$, covering incidence angles between $18^{\circ}$ and $59^{\circ}$ and are, therefore, colocated to the SAR imagettes. From these triplets, wind speed, and wind direction were derived by the Centere ERS d'Archivage et de Traitement, using the C-band model CMOD_IFR2 with an

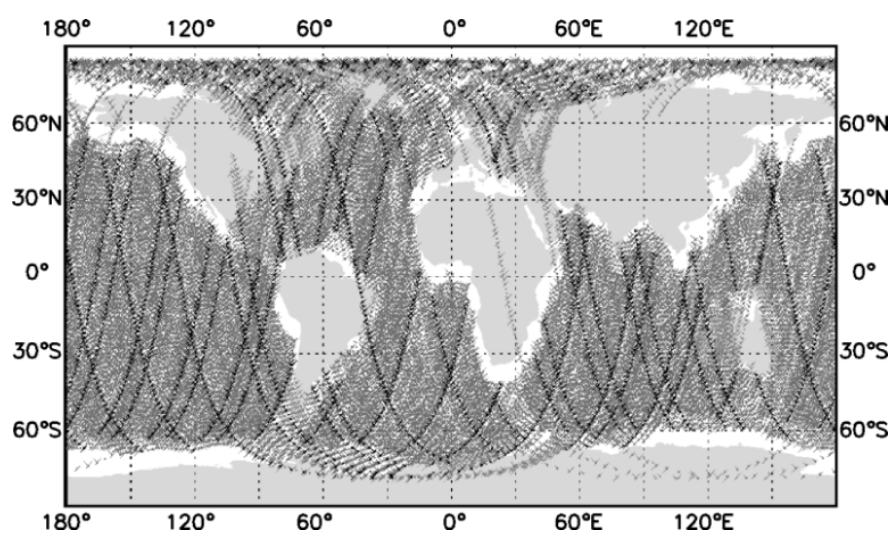

Fig. 1. Global distribution of the 27 days of ERS-2 SAR wave mode imagettes. The black stars represent the coverage of one day.

accuracy of $1.2 \mathrm{~ms}^{-1}$ in wind speed and $15^{\circ}$ in wind direction [20].

In addition to the ERS-2 SAR and SCAT data, colocated wind data from the atmospheric model of ECMWF were available. The model version used in 1996 was T213L31 and uses a spectral formulation in the horizontal, with triangular truncation at total wavenumber 213 . In the vertical, 31 levels are considered between the earth surface and $30 \mathrm{~km}$ height, and the lower levels are terrain-following. The model produces global ECMWF wind fields (ECMWF forecast) for the four main synoptic hours $00,06,12$, and $18 \mathrm{UTC}$ with a grid size of $0.5^{\circ}$. In a 6-h period, several observational data, e.g., ERS-2 SCAT, aircraft, and in situ, of several parameters, e.g., wind, temperature and surface pressure, are assimilated into the model and give the routine ECMWF analysis wind fields.

\section{DEPENDENCY OF SAR INTENSITY ON WIND}

At moderate incidence angles between $20^{\circ}$ and $70^{\circ}$ the backscatter measured by the SAR is dominated by the backscatter from centimeter-scale surface roughness, which is in resonance with the incidence radiation of the radar, the Bragg scattering. For the range of incidence angles covered by the ERS-2 SAR wave mode $\left(22.7^{\circ}\right.$ to $\left.23.4^{\circ}\right)$, the range of scattering roughness lengths is strongly influenced by the local wind field and, therefore, allows the backscatter to be a measure of the local wind.

It is well known that the wind speed can only be retrieved from SAR data that are free of sea surface features not due to the local wind, e.g., sea ice and slicks (Fig. 2). To exclude SAR imagettes that contain features not associated with the local wind, a filter is applied. The filter was developed by Lehner et al. [21] and distinguishes between homogeneous and inhomogeneous SAR imagettes. The technique was originally proposed to test the homogeneity of ocean wave fields, e.g., the shift invariance of the ocean wave spectrum. Inhomogeneous SAR imagettes are significantly affected by features such as surface slicks and sea ice. The technique is based on a test of statistical properties of periodograms, which are commonly used for spectral estimation. According to standard theory, spectral densities estimated from a single periodogram are negative exponentially distributed [22]. A standard approach to reduce the variance of the spectral estimator is to average periodograms estimated 


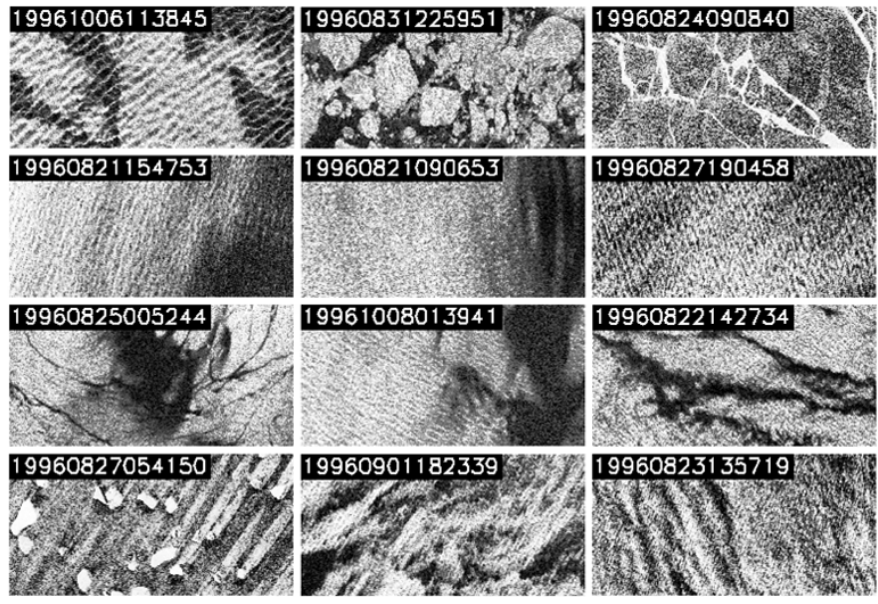

Fig. 2. Typical examples of strongly inhomogeneous SAR imagettes that are affected by sea ice, atmospheric effects, slicks, and other features (from top to bottom row).

from subimages. To check the homogeneity of SAR imagettes 32 subimages of about $1 \mathrm{~km} \times 1 \mathrm{~km}$ size are used to estimate the mean and variance of the periodogram $P$. The estimator $\vartheta$ of the inhomogeneity test is defined as

$$
\vartheta=\frac{\sum_{k} \frac{\overline{\operatorname{var}}\left(P_{k}\right)}{\operatorname{mean}\left(P_{k}\right)}}{\sum_{k} \overline{\operatorname{mean}}\left(P_{k}\right)}
$$

where $k$ is the wavenumber and $\overline{\text { mean }}$ and $\overline{\text { var }}$ are standard estimators for the periodogram mean and variance. For a perfectly homogeneous image, the inhomogeneity parameter $\vartheta$ should be 1. However, visual inspection of the SAR imagettes resulted in a choice of $\vartheta=1.05$ as threshold. Therefore, in the following, all SAR imagettes with a inhomogeneity parameter $\vartheta \geq 1.05$ were defined as inhomogeneous and were omitted from this analysis.

To assess the performance of the inhomogeneity test, a visual inspection of the SAR imagettes was carried out to detect errors of the first kind (a homogeneous imagette is classified as inhomogeneous) and the second kind (an inhomogeneous imagette is classified as homogeneous). Concerning the threshold of 1.05 for the parameter $\vartheta, 6.2 \%$ of the SAR imagettes did not pass the inhomogeneity test. Visual inspection of the inhomogenous SAR imagettes resulted in $93 \%$ of these imagettes showing artifacts, e.g., sea ice and slicks, which cannot be associated to the wind. This means that the error of first kind occurs with a probability of 7\%. Among the SAR imagettes, which are classified as homogeneous, $6.9 \%$ are not suited for wind measurements, suggesting that the errors of the first and the second kind have the same order of probability. In $60 \%$ of the cases with misclassifications of the second kind, homogeneous sea ice features affect the SAR imagettes. The most straightforward way to deal with the latter cases is to exclude the polar regions. It is clear that, in addition to the inhomogeneity test, other methods have to be developed to distinguish SAR imagettes acquired over ice from those over open water. In Fig. 3, wind speeds from the ERS-2 SCAT are plotted against mean SAR imagette intensities considering all homogenous imagettes. The correlation considering all SAR imagettes is 0.73 ; neglecting the inhomogenous imagettes,

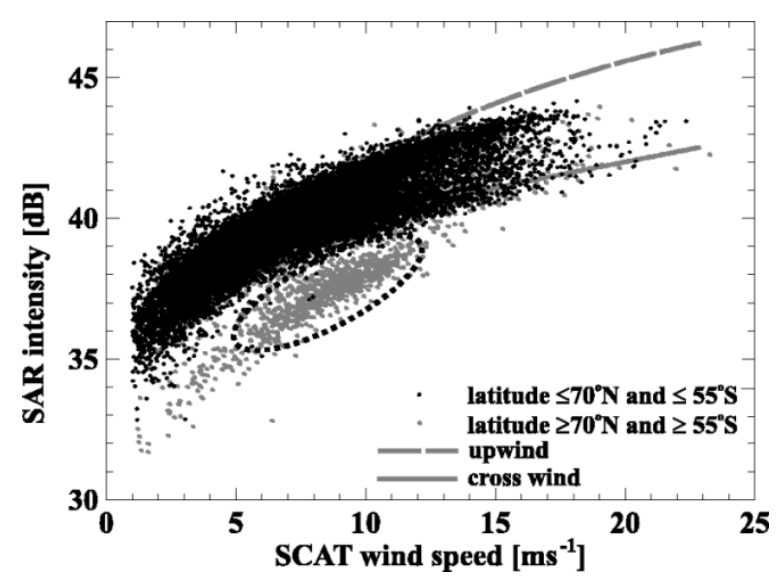

Fig. 3. Wind speeds from ERS-2 SCAT versus mean SAR imagette intensities. The black dots represent SAR imagettes between $55^{\circ} \mathrm{S}$ and $70^{\circ} \mathrm{N}$ and the light gray ones imagettes beyond $55^{\circ} \mathrm{S}$ and $70^{\circ} \mathrm{N}$, respectively. Superimposed are the curves resulting from the $\mathrm{C}$-band SCAT model CMOD4 at an incidence angle of $23^{\circ}$ for upwind (dashed line) and cross wind (solid line). The CMOD4 curves were shifted by $45.5 \mathrm{~dB}$.

the correlation improves to 0.79 , and, if in addition, all imagettes beyond $70^{\circ} \mathrm{N}$ and $55^{\circ} \mathrm{S}$ are neglected, the correlation is 0.86 . In the scatter plot of Fig. 3, all light gray dots represent SAR imagettes beyond $70^{\circ} \mathrm{N}$ and $55^{\circ} \mathrm{S}$. It is obvious that most of the outliers are located in latitudes where sea ice is very likely, and these outliers are responsible for the low correlation of 0.79 .

To investigate the dependency of the SAR imagette intensity on wind speed, the mean imagette intensity is derived and compared to the colocated wind from the SCAT aboard ERS-2. In Fig. 3, wind speeds from the ERS-2 SCAT are plotted against mean SAR imagette intensities. The correlation considering all SAR imagettes is 0.79 , while 0.86 is achieved when neglecting SAR imagettes beyond $70^{\circ} \mathrm{N}$ and $55^{\circ} \mathrm{S}$. The encircled outliers in Fig. 3 are located in latitudes where sea ice is very likely and are responsible for the low correlation of 0.79 . For comparison, results of the empirical C-band SCAT model CMOD4 [10], which describes the dependency of the NRCS on the wind, is used. CMOD4 was especially tuned to the ERS SCATs and has been shown to be accurate to $1.65 \mathrm{~ms}^{-1}$ in wind speed and $16.7^{\circ}$ in wind direction [10]. However, investigations by Quilfen et al. [11] and Donnelly et al. [23] have shown that CMOD4 significantly underestimates the wind speed at high wind speeds above $20 \mathrm{~ms}^{-1}$.

In Fig. 3, the CMOD4 curves for upwind (toward the radar look direction) and cross wind (perpendicular to radar look direction) at an incidence angle of $23^{\circ}$ are superimposed. They give a reference for the dependency of radar backscatter on the wind. To fit the SAR imagette intensities, the CMOD4 curves were shifted by $45.5 \mathrm{~dB}$. The shift of $45.5 \mathrm{~dB}$ represents the bias to the CMOD4-retrieved NRCS and gives a rough estimate of the calibration constant (for details refer to Section IV). Due to the nonlinear relation between wind speed and the NRCS and the additional dependency of NRCS on wind direction, indicated by the CMOD4 curves, a much higher correlation cannot be expected. However, it is obvious that the CMOD4 curves are in good agreement with the dependency of SAR intensity on wind speed. 

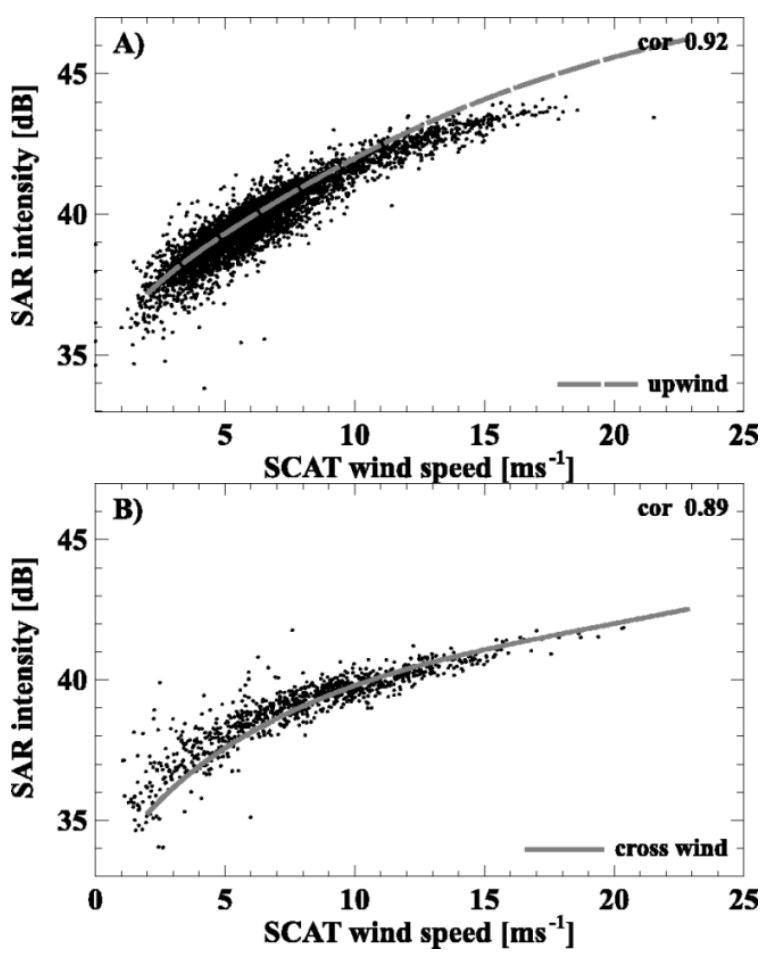

Fig. 4. Wind speeds from ERS-2 SCAT versus mean SAR imagette intensities. The black dots represent SAR imagettes between $55^{\circ} \mathrm{S}$ and $70^{\circ} \mathrm{N}$ considering SCAT wind directions of $\pm 10^{\circ}$ up- and downwind (a) and $\pm 10^{\circ}$ cross wind (b).

To investigate the dependency of SAR imagette intensity on wind speed and direction, the wind directions from the colocated ERS-2 SCAT data were partitioned into $20^{\circ}$ intervals. In Fig. 4, wind speeds from ERS-2 SCAT are plotted versus mean SAR imagette intensities considering SCAT wind directions of $\pm 10^{\circ}$ from up- and downwind [Fig. 4(A)] and from cross wind [Fig. 4(B)], respectively. Comparing these plots to the one shown in Fig. 3 indicates a significantly better correlation for both cases. The cross wind case has a lower correlation than the up- and downwind case, which is due to the stronger nonlinearity of the wind speed intensity relation for cross winds than for up- and downwind, as indicated by the CMOD4 curves.

In case of up- and downwind, it is obvious that for SAR imagette intensities over $42 \mathrm{~dB}$ the CMOD4 predicted intensities are significantly higher than the SAR measured intensities [Fig. 4(A)]. This also corresponds to distinct differences at upand downwind between CMOD4 and SAR imagette intensities at wind speeds above $10 \mathrm{~ms}^{-1}$. In the crosswind case, no characteristic differences between CMOD4 and SAR imagette intensities are apparent, and there is an overall good agreement for wind speeds up to $20 \mathrm{~ms}^{-1}$. This indicates that the disagreement between COMD4 and SAR imagette intensities is predominantly caused by the choice of the analogue-to-digital converter (ADC) settings of the SAR system and not by uncertainties of the CMOD4 at high wind speeds. The correct choice of ADC settings is a well-known problem affecting ERS- 1 and ERS-2 SAR images in the case of high radar backscatter [1], [24]. When radar backscatter is high, the input power to the $\mathrm{ADC}$ is too high, which leads to saturation of the ADC and in turn to an output power of the ADC that is lower than the input power. In the other case, when radar backscatter is very low, below the offset applied to the ADC, the digitized power is higher than the input power to the ADC [25]. In the following, these ADC errors are referred to as power loss. This later effect can be seen especially for wind directions of $\pm 10^{\circ}$ cross wind at wind speeds below $6 \mathrm{~ms}^{-1}$ [Fig. 4(B)], where the SAR intensity is higher than that predicted by CMOD4. Contrary to the five-bit sampling in the ERS-2 SAR image mode, the wave mode data are only four-bit sampled, which leads to a distinct increase of power-loss errors requiring correction. Nevertheless, a strong wind dependency of SAR imagette intensity is observed and is used in the following sections to retrieve wind speeds applying the CMOD4 model as well as NNs.

\section{SAR WIND RETRIEVAL USING CMOD4}

So far, in contrast to the image mode, SAR imagettes are not calibrated and there is no calibration information available, such as a calibration constant, a power-loss lookup table, or an antenna pattern. However, to apply C-band models to SAR imagettes, their mean intensity has to be accurately calibrated and transformed to the NRCS. In the following, the NRCS is derived from the SAR imagettes according to the scheme for ERS-1 and ERS-2 SAR image mode data proposed by Meadows $\mathrm{et}$ al. [24]. Most of the parameters that have to be applied are incidence-angle dependent; however, in case of SAR imagettes, the dependence on incidence angle can be neglected due to the small range of incidence angles $\left(\sim 0.7^{\circ}\right)$, which leads only to minor changes in the calibration constant and antenna pattern. Therefore, the NRCS $\left(\sigma_{0}\right)$ can be approximated for SAR imagettes very well by the following equation:

$$
\sigma_{0}=A^{2} \cdot k_{\mathrm{im}} \cdot \mathrm{pl}_{\mathrm{im}}
$$

where $A$ is the amplitude, $k_{\text {im }}$ a calibration constant for ERS-2 SAR imagettes including the range spreading loss, and $\mathrm{pl}_{\mathrm{im}}$ the power loss corresponding to the correction for ADC errors.

To determine $k_{\mathrm{im}}$, typically measurements from transponders are analyzed that are not available for the investigated SAR imagettes. Therefore, in the following, calibration is performed by applying a new efficient method, based on knowledge of the dependency of the NRCS on the wind. Given the wind vector, CMOD4 allows an estimation of the NRCS for each SAR imagette. Therefore, the wind speed and wind direction information from the colocated ECMWF model or ERS-2 SCAT data and a fixed incidence angle of $23^{\circ}$ are taken as input to the CMOD4. Computing the bias between SAR imagette intensities and colocated CMOD4-retrieved NRCSs results in an estimate of $k_{\mathrm{im}}$. To obtain an accurate estimate of $k_{\mathrm{im}}$, only SAR imagettes in the range of 5-8 $\mathrm{ms}^{-1}$ in wind speed are considered so as to exclude SAR imagettes affected by power-loss errors caused by the ADC. This procedure is followed using CMOD4, together with each of the wind datasets (ECMWF model and ERS-2 SCAT wind data). The mean resulting $k_{\mathrm{im}}$ is equal to $-44.96 \mathrm{~dB}$ with differences of $<0.1 \mathrm{~dB}$ between each dataset, which is in the order of the radiometric accuracy of ERS-2 SAR.

To check the consistency of the retrieved calibration constant, the NRCS is derived for each SAR imagette neglecting the power loss and is used to derive the wind speed via CMOD4. Therefore, the SAR imagette NRCS, at a fixed incidence 

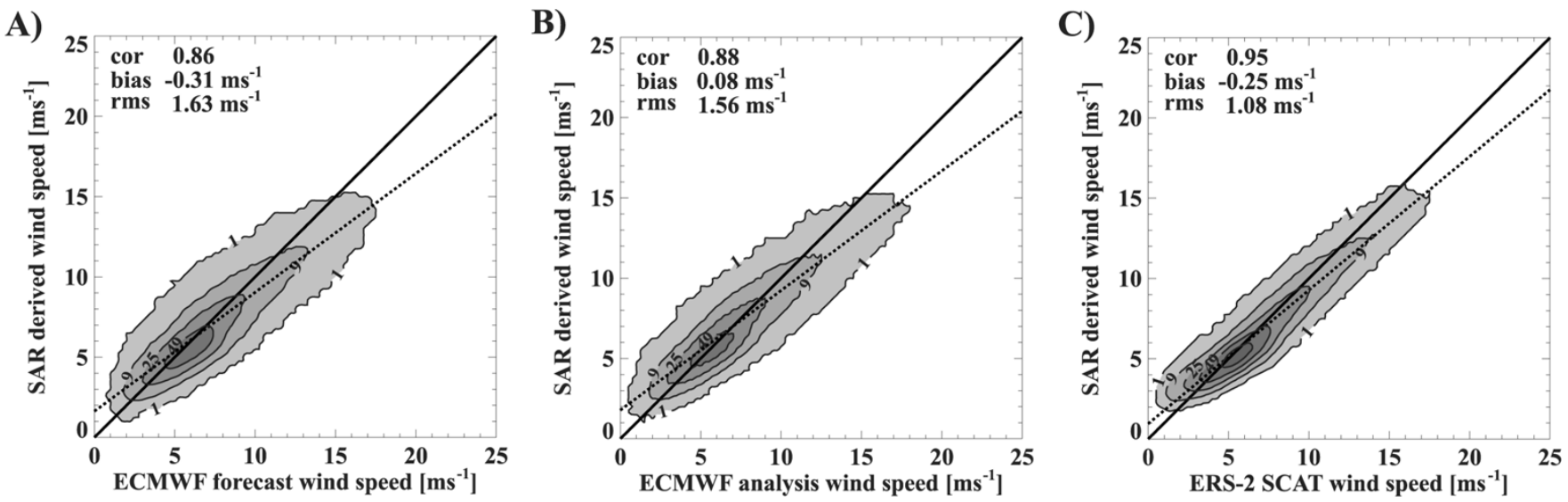

Fig. 5. (a) Wind speeds from ECMWF forecast, (b) ECMWF analysis, and (c) ERS-2 SCAT versus wind speeds retrieved from SAR imagette-derived NRCS neglecting power-loss corrections. The dotted curve gives the regression line.

angle of $23^{\circ}$ and the wind direction of the colocated ECMWF model or ERS-2 SCAT data, is taken as input to the CMOD4. The resulting comparisons are plotted in Fig. 5, where the wind speeds from the ECMWF forecast [Fig. 5(A)], ECMWF analysis [Fig. 5(B)], and ERS-2 SCAT [Fig. 5(C)] are plotted versus the SAR imagette-derived wind speeds. The corresponding main statistical parameters are given in the upper left of each scatterplot. There are only minor differences between the statistics considering the ECMWF forecast and ECMWF analysis. However, comparison of SAR imagette-retrieved wind speeds to ERS-2-SCAT-retrieved wind speeds show a significantly better correlation than the comparison to ECMWF model results.

The overall good agreement between the SAR and SCAT-retrieved wind speeds is expected. The low bias is due to the calibration method, which ensures, by construction, that the mean SAR-derived wind speed must equal the mean SCAT wind speed in the interval of $5-8 \mathrm{~ms}^{-1}$. Furthermore, the good agreement is due to the similarity of the two sensors and the colocation in space and time, as well as the fact that both instruments use a similar C-band model to retrieve the wind speed. However, it is obvious that with increasing wind speed SAR-derived wind speeds underestimate the wind speed significantly. This effect is due to power loss caused by saturation of the ADC. In Fig. 5(C) a slight overestimation for SAR imagette-retrieved wind speeds can be seen below $5 \mathrm{~ms}^{-1}$, which is due to the power gain caused by bit redundancy at the ADC.

The power-loss correction can be estimated in a manner similar to that of the calibration constant. Therefore, the power-loss-affected NRCSs $\sigma_{p l}$ of all SAR imagettes are derived according to

$$
\sigma_{p l}=A^{2} \cdot k_{\mathrm{im}} .
$$

The expected NRCS of each SAR imagette was retrieved by applying the CMOD4 with input of a fixed incidence angle $\left(23^{\circ}\right)$ and the wind speed and direction from the colocated ECMWF model or ERS-2 SCAT data. The differences between the expected and power-loss-affected NRCS give an estimate of the power loss for each SAR imagette. To obtain a function of the power loss, a third-degree polynomial was fitted to

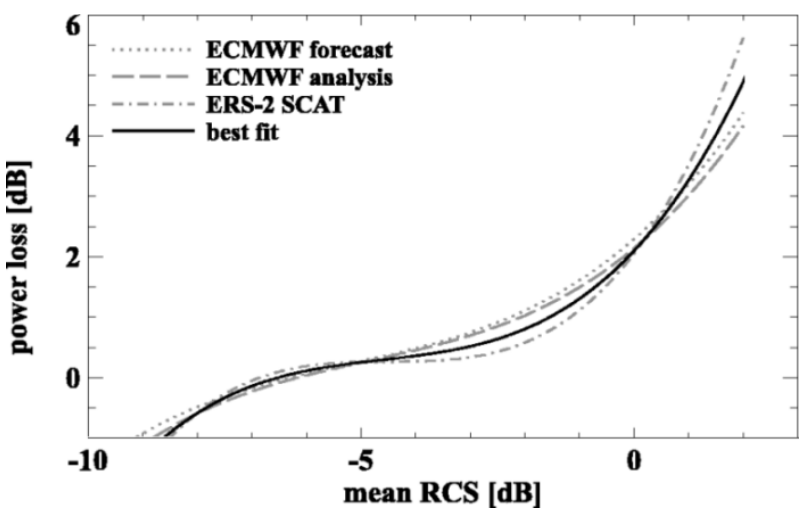

Fig. 6. Mean radar cross section versus power-loss correction.

the differences between the expected and power-loss-affected NRCSs. In Fig. 6, the mean radar cross section is plotted against the power-loss correction resulting from each dataset together with the best fit considering all data. The power-loss correction curves look very similar to the power-loss corrections, which are applied to the ERS-2 SAR data in the imaging mode. However, for increasing NRCS, the estimated power-loss corrections are of low accuracy, due to the lack of data available at high wind speeds, which leads to high NRCSs.

To check the consistency of the complete calibration, again wind speeds is retrieved from SAR imagette NRCSs according to (2). Again wind speeds are derived using CMOD4 and a fixed incidence angle of $23^{\circ}$ and the wind directions according to the colocated ECMWF model or ERS-2 SCAT data. The comparisons of wind speeds resulting from SAR imagettes to the colocated ECMWF model and ERS-2 SCAT wind speeds are given in Fig. 7. Again, the statistical parameters are similar considering the winds from ECMWF forecast and ECMWF analysis and are significantly better when comparing to ERS-2 SCAT measurements. The scatter in the comparison of ERS-2 SAR imagette and SCAT-retrieved wind speeds is expected because a SAR imagette covers only $2.5 \%$ of the area of a SCAT resolution cell. It is obvious that consideration of the power loss significantly improves the consistency between SAR imagette and SCAT-retrieved wind speeds at high wind speeds. This can be seen by the regression lines, which are closer to the diagonal 
A)

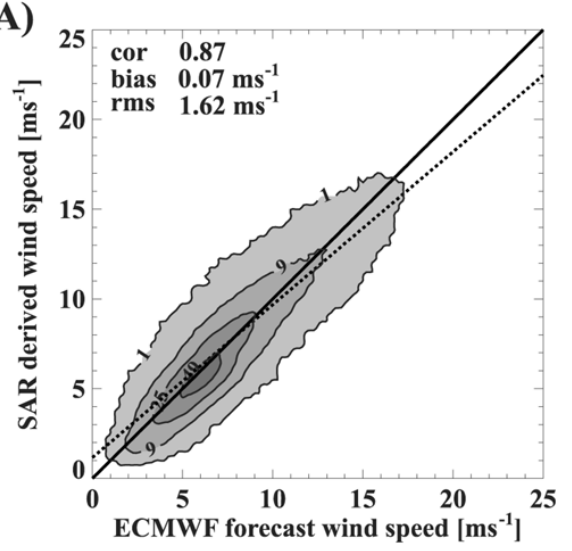

B)

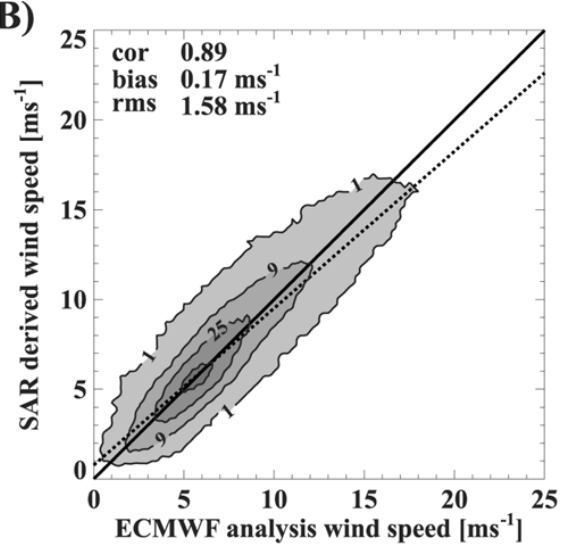

C)

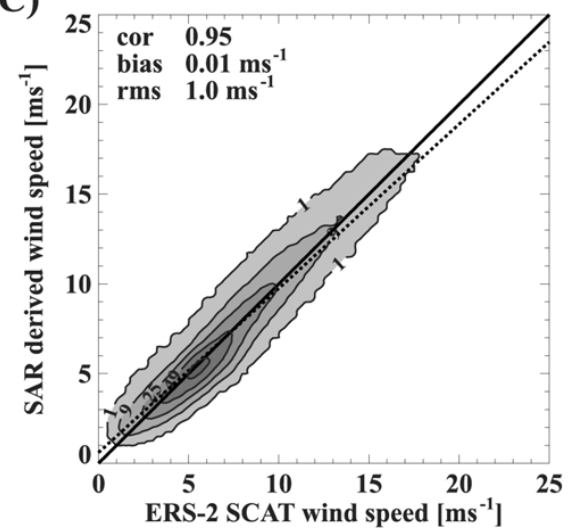

Fig. 7. Same as Fig. 5 but considering power-loss corrections.

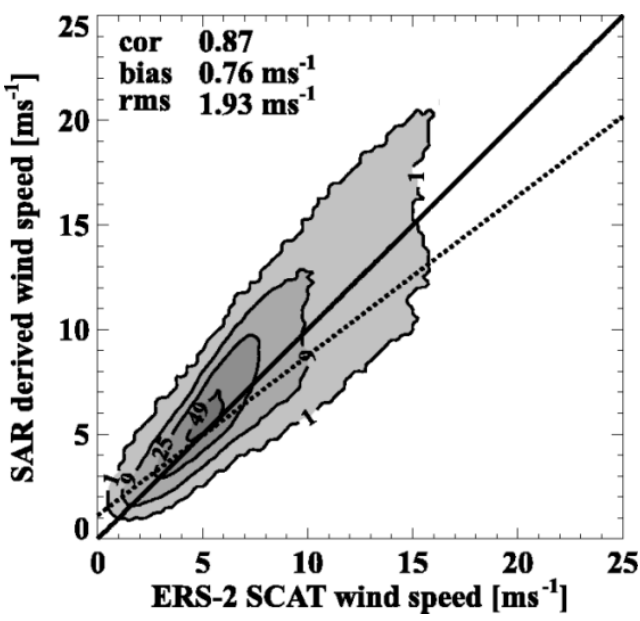

Fig. 8. Scatterplot of ERS-2 SCAT wind speeds versus SAR imagetteretrieved wind speeds using the CMOD4 model with a fixed wind direction of $45^{\circ}$. The dotted curve gives the regression line.

representing the optimum regression line than in Fig. 5. However, the change in the main statistical parameters is negligible. To obtain a more accurate correction of the ADC errors, especially at high NRCS $(\sim 43 \mathrm{~dB})$, the SAR imagette raw data have to be analyzed according to the method described by Meadows et al. [25].

In the previous analysis, wind direction information was taken from the ECMWF model or ERS-2 SCAT data. However, in case there is no wind direction information available, the wind direction has to be set to a constant. In Fig. 8, wind speeds from ERS-2 SCAT are plotted against the results from SAR imagettes wind speeds, which are derived by applying CMOD4 to the fully calibrated imagettes with a fixed wind direction of $45^{\circ}$, the optimal choice. The dependency of wind direction increases significantly with wind speed, which is the reason for the large scatter for wind speeds above $10 \mathrm{~ms}^{-1}$. In Fig. 9, the rms errors of the comparisons of SAR imagette-retrieved wind speeds to ERS-2 SCAT wind speeds is plotted for wind speed intervals of $2 \mathrm{~ms}^{-1}$ between 0 and $22 \mathrm{~ms}^{-1}$. Assuming a fixed wind direction for wind speed retrieval results in an rms error of $\leq 2.1 \mathrm{~ms}^{-1}$ for wind speeds below $10 \mathrm{~ms}^{-1}$ and the rms error increases significantly for wind speeds above $8 \mathrm{~ms}^{-1}$. If the wind direction is considered, the rms error is $\leq 1.7 \mathrm{~ms}^{-1}$ for

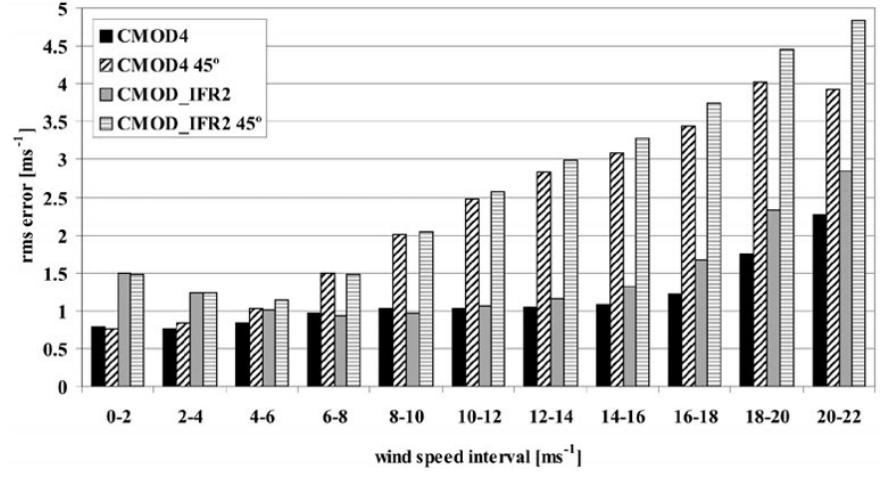

Fig. 9. Bar plot of rms errors of wind speed from the comparison of SAR imagette wind speeds to ERS-2 SCAT wind speeds. The rms errors were derived for wind speed intervals of $2 \mathrm{~ms}^{-1}$.

wind speeds below $18 \mathrm{~ms}^{-1}$ and then increases significantly, which is due to the lack of wind data available at high wind speeds for the SAR imagette calibration.

\section{SAR WIND RETRIEVAL USING NNs}

A straightforward method for retrieving wind speeds from uncalibrated SAR imagettes can be obtained by using NNs, which allow for the retrieval of the wind speed directly from the SAR imagette intensity, independent of the knowledge of the NRCS. The NN approach does not require explicit models for the SAR imaging process and can, therefore, be easily applied to any system configuration, i.e., polarization, incidence angle, etc. The only requirements for application of an NN to SAR-wind-speed retrieval is the relative radiometric stability of the SAR system as well as a dependency of backscatter on wind speed. In case of the ERS SAR systems both requirements are fulfilled. In the following, NNs are used as a multiple nonlinear regression technique to parameterize the relationship between the SAR imagette intensity and ocean surface wind.

An NN is built up of several layers: an input layer, one or more hidden layers and one output layer. Each layer consists of "neurons"; the input layer has as many neurons as input parameters and the output layer as many neurons as output parameters. The number of neurons in the hidden layer(s) is dependent upon the problem. Each neuron in a layer is linked to each neuron of the neighboring layer with a weight. 


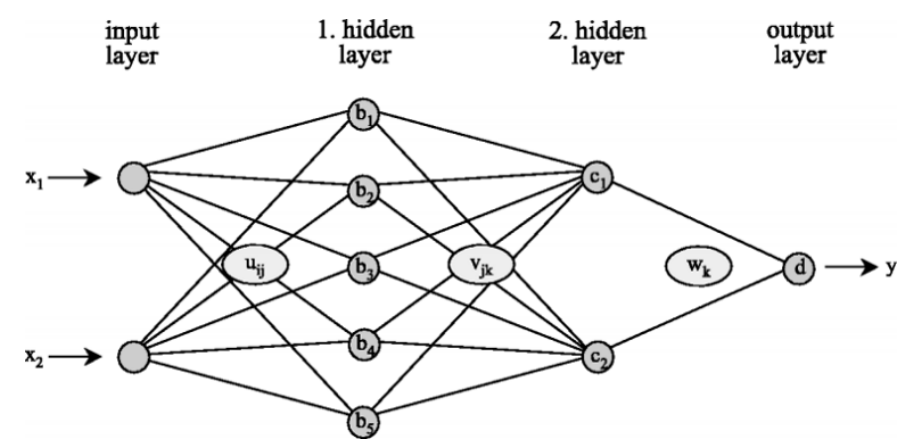

Fig. 10. Schematic of a neural network having two input parameters, two hidden layers with five, and two neurons and an output layer with one parameter.

An example of an NN with two hidden layers is given in Fig. 10. The output value $N_{\text {out }}$ of each neuron is derived according to

$$
N_{\text {out }}=S\left(-N_{\text {bias }}+\sum_{i=1}^{n} w_{i} x_{i}\right)
$$

where $N_{\text {bias }}$ is a bias value specific to each neuron; $n$ is the number of incoming links; $w$ is a weight specific to each neuron; $x$ is the output value of the neuron in the preceding layer; and $S$ is a nonlinear function assuming monotonically increasing values between zero and one as the value of the argument goes from $-\infty$ to $\infty$. The most common choice, also used in the NNs applied here, is the function $[1+\exp (-x)]^{-1}$. An NN operates sequentially from layer to layer; output neurons of the first layer are given by the input values. The output of each neuron of the first hidden layer is computed by summation of the weighted inputs, shifting by the bias and application of the nonlinear function. This is repeated for each layer until the output layer is reached, giving the results of the NN. To determine an NN, a sufficiently large set of input and output vectors has to be available to generate a training and a test sample. During the training of the $\mathrm{NN}$, the values of the biases and weights are changed to minimize the error function. The resulting trained $\mathrm{NN}$ has to be tested with the test sample for its generalization power, e.g., whether reasonable results are produced for input values, which are not included in the training sample.

Two NNs are trained, one using the mean SAR imagette intensity together with the colocated ERS-2 SCAT wind speed and a second considering ERS-2 SCAT wind direction in addition. For both cases, the training sample consisted of $\sim 12000$ colocations, which are selected randomly. The remaining $\sim 11600$ colocations are taken to test the trained NN. The first $\mathrm{NN}$, considering only SAR imagette intensity, is composed of three hidden layers with eight neurons in the first hidden layer, five in the second, and two in the third. The second NN is also composed of three hidden layers, however, with six neurons in the first, four in the second, and two in the third. In Fig. 11 wind speeds from the ERS-2 SCAT are plotted against the wind speed resulting from SAR imagettes using the first NN 1) considering intensity and the second $N N 2$ ) considering wind directions in addition. The corresponding statistics of the comparison are given in the upper left of the scatter plots. The main statistical parameters for comparisons of ECMWF model
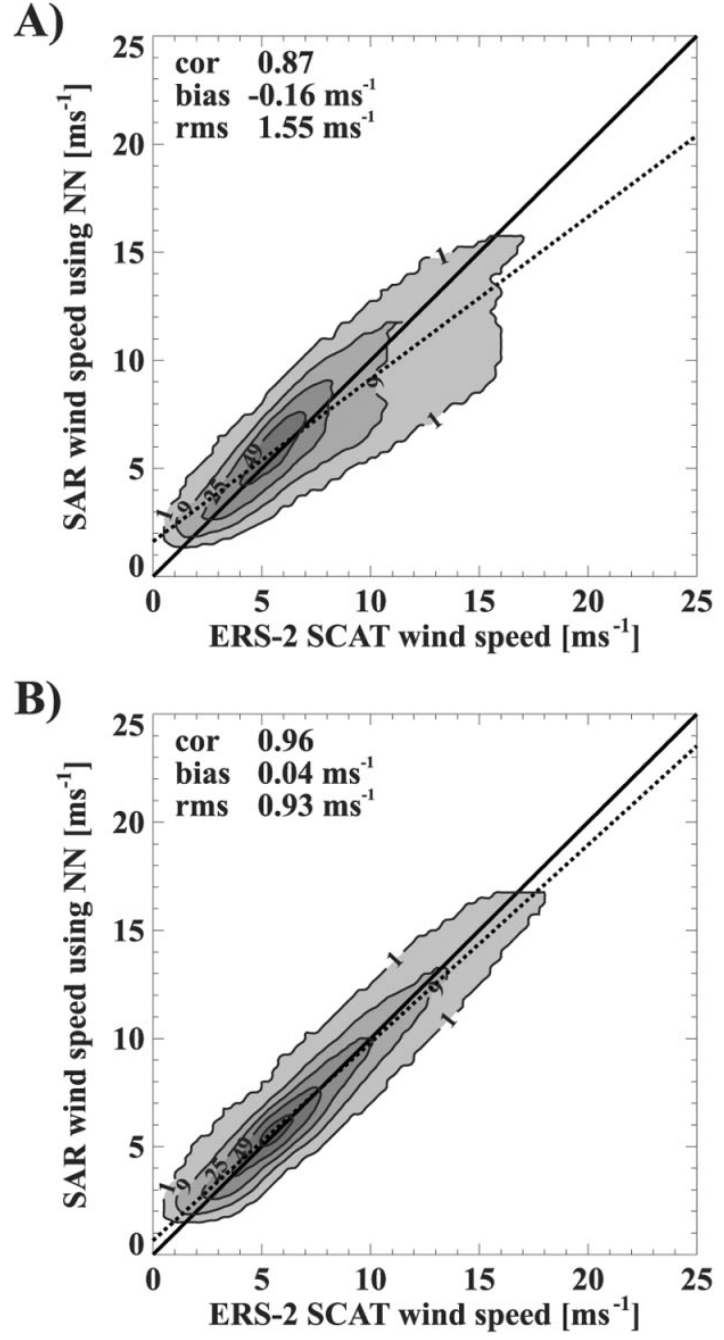

Fig. 11. Scatterplots giving the comparison of the ERS-2 SCAT wind speeds versus wind speeds retrieved from collocated SAR imagettes. The SAR imagette wind speeds were retrieved using an NN with the SAR imagette intensity and the wind direction from the collocated ERS-2 SCAT data as input. The dotted curve gives the regression line.

TABLE I

MAIN STATISTICAL PARAMETERS FOR COMPARISON OF SAR-IMAGETTERETRIEVED WIND SPEEDS TO ECMWF MODEL AND ERS-2 SCAT WIND SPEEDS USING A NN WITH IMAGETTE INTENSITY AS INPUT (FIRST NN) AND WiTH ERS-2 SCAT WIND DIRECTIONS IN ADDITION AS INPUT (SECOND NN)

\begin{tabular}{|c|c|c|c|}
\hline $\begin{array}{l}\text { Statistics of } \\
\text { first NN }\end{array}$ & $\begin{array}{c}\text { ECMWF } \\
\text { forecast }\end{array}$ & $\begin{array}{c}\text { ECMWF } \\
\text { analysis }\end{array}$ & $\begin{array}{l}\text { ERS-2 } \\
\text { SCAT }\end{array}$ \\
\hline cor & 0.83 & 0.84 & 0.87 \\
\hline bias $\left[\mathrm{ms}^{-1}\right]$ & -0.57 & -0.39 & -0.16 \\
\hline $\mathrm{rms}\left[\mathrm{ms}^{-1}\right]$ & 1.87 & 1.83 & 1.55 \\
\hline nr. of coloc. & 19995 & 17636 & 19284 \\
\hline $\begin{array}{l}\text { Statistics of } \\
\text { second NN }\end{array}$ & $\begin{array}{c}\text { ECMWF } \\
\text { forecast }\end{array}$ & $\begin{array}{c}\text { ECMWF } \\
\text { analysis }\end{array}$ & $\begin{array}{l}\text { ERS-2 } \\
\text { SCAT }\end{array}$ \\
\hline cor & 0.89 & 0.88 & 0.96 \\
\hline bias $\left[\mathrm{ms}^{-1}\right]$ & 0.13 & 0.09 & 0.04 \\
\hline $\mathrm{rms}\left[\mathrm{ms}^{-1}\right]$ & 1.5 & 1.53 & 0.93 \\
\hline nr. of coloc. & 19995 & 17636 & 19284 \\
\hline
\end{tabular}

and ERS-2 SCAT wind speeds to SAR imagette-retrieved wind speeds via both NNs are given in Table I. 


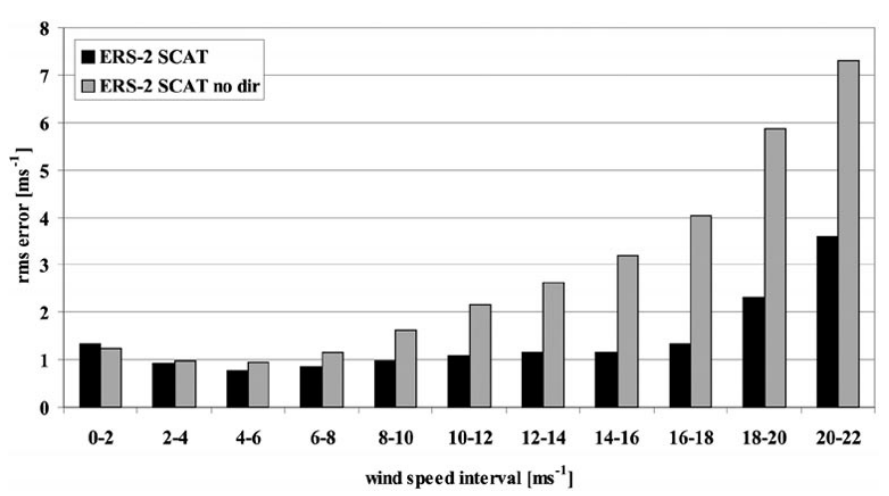

Fig. 12. Bar plot as given in Fig. 9. SAR imagette wind speeds were retrieved using the NN considering mean SAR imagette intensity and wind direction from the ERS-2 SCAT.

Although the wind direction is not considered in the first NN, the statistics are quite good. As anticipated and similar to Fig. 8, the scatter increases significantly with wind speed, due to the additional dependency of SAR imagette intensity on wind direction. Considering wind direction significantly decreases the scatter over the range of mid to high wind speeds resulting in a correlation of 0.96 , a bias of $0.04 \mathrm{~ms}^{-1}$ and an rms of $0.93 \mathrm{~ms}^{-1}$.

In Fig. 12, the rms errors in wind speed of the comparisons of SAR imagette-retrieved wind speeds to ERS-2 SCAT wind speeds is plotted for intervalls of $2 \mathrm{~ms}^{-1}$. For SAR imagette wind speeds that are retrieved using the NN considering mean SAR imagette intensity and wind direction from the ERS-2 SCAT, the rms error is rather constant for wind speeds below $18 \mathrm{~ms}^{-1}$. Above $18 \mathrm{~ms}^{-1}$, the error increases significantly, due to the insufficient number of available data at high wind speeds for the training of NNs. In the case of SAR imagette-retrieved wind speeds using the NN with only input of mean SAR imagette intensity, the error increases significantly for wind speeds above $10 \mathrm{~ms}^{-1}$, showing that in addition wind directions have to be taken into account.

Again the statistics of each of the colocated datasets differ significantly. Comparison of wind speeds from SAR imagettes and ERS-2 SCAT shows the best consistency, as expected, due to the similarity of the two instruments. Also, the better consistency of SAR imagette-retrieved wind speeds to the results from the ECMWF analysis than to the ECMWF forecast is as expected. However, although additional sources of data are considered in the ECMWF analysis, the results are not significantly better in comparison to SAR imagette wind speeds than those of the ECMWF forecast [see also Fig. 7(A) and (B)].

Comparing the resulting correlation, bias, and root mean square error achieved by using the CMOD4 (Fig. 7) to those obtained by using an NN for SAR imagette wind speed retrieval look very similar. However, in all cases the $\mathrm{NN}$-retrieved wind speeds give slightly better results, which is to be expected as there is no separate calibration step to be performed.

\section{Discussion of MAIN DifFERENCES IN Wind SPEED}

To analyze the geographical distribution and sources of differences between wind speed from ERS-2 SAR and the ECMWF forecast, ECMWF analysis and ERS-2 SCAT data,

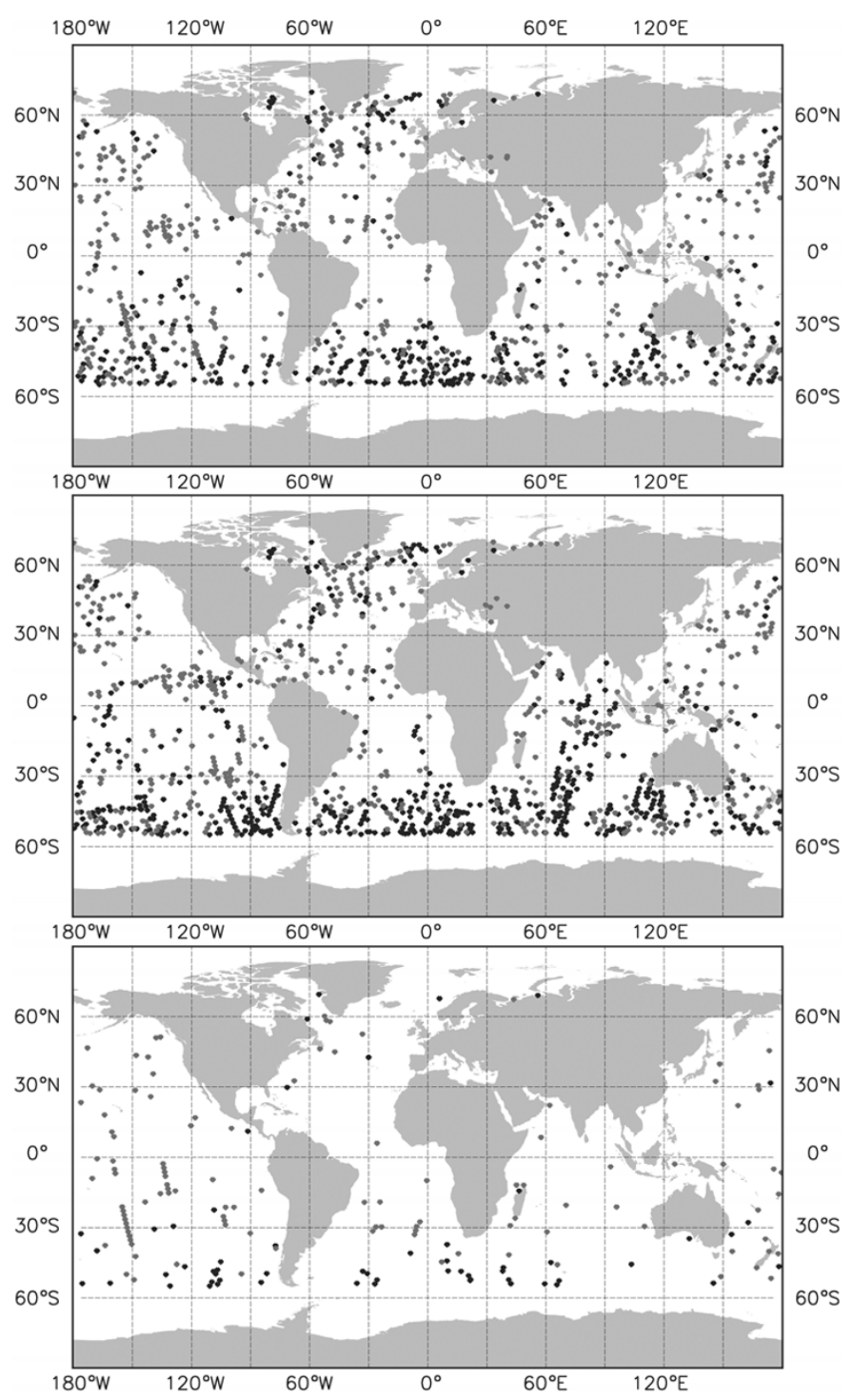

Fig. 13. Geographical locations of SAR imagettes that differ in wind speed by $\geq 3 \mathrm{~ms}^{-1}$ from (top) the ECMWF forecast, (middle) ECMWF analysis, and (bottom) ERS-2 SCAT data. Locations marked by gray symbols represent higher wind speeds for SAR imagettes and black symbols a lower wind speed.

the locations of the differences are marked on a world map. In Fig. 13 the geographical locations are marked where the differences in wind speed between the SAR imagettes and the ECMWF forecast, ECMWF analysis and ERS-2 SCAT (from top to bottom) are $\geq 3 \mathrm{~ms}^{-1}$. In all plots of Fig. 13, most of the wind speed differences are located in the Southern Hemisphere at high latitudes (above $30^{\circ} \mathrm{S}$ ), where the ECMWF models and ERS-2 SCAT predict higher wind speeds than the wind speeds measured by ERS-2 SAR. Furthermore, most of the underestimated SAR winds occurred at high wind speeds (above $16 \mathrm{~ms}^{-1}$ ), which occur more often at higher southern latitudes. This underestimation at high winds speeds is due to the low number of colocated data available at high wind speeds for the training of the NNs and the poor performance of NNs for extrapolation. In the comparison to ERS-2 SCAT data, wind-speed differences of $\pm 3 \mathrm{~ms}^{-1}$ occur significantly less, which again is expected due to the similarity of the instruments and exact colocation in time. 


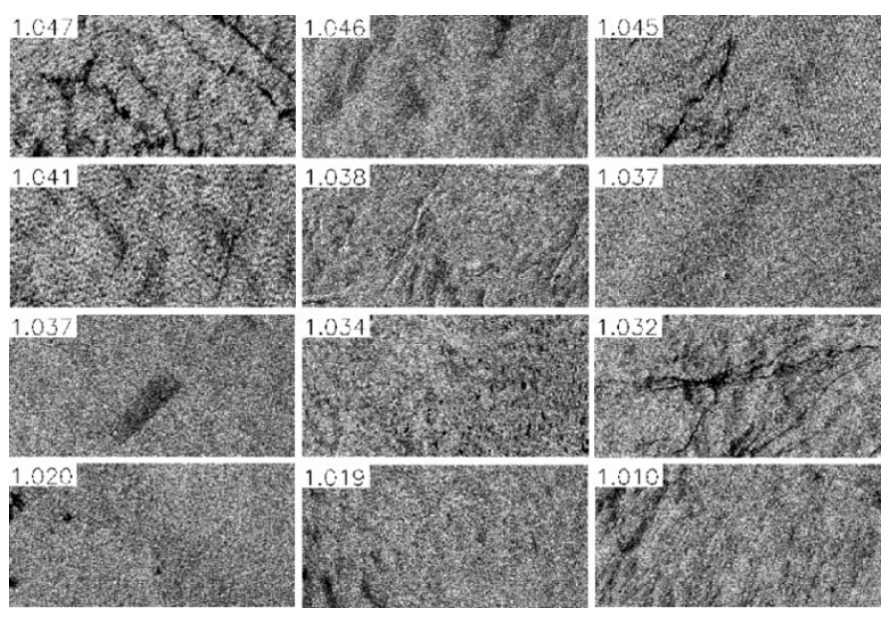

Fig. 14. Typical SAR imagettes where the differences in wind speed are $\geq$ $4 \mathrm{~ms}^{-1}$ in comparison to the wind speed measured by the colocated ECMWF model analysis.

In contrast to most other radar sensors for wind-speed retrieval, e.g., altimeter and SCAT, the SAR is an imaging radar that gives an image of the ocean surface. This is one of the major advantages of SAR imagettes for wind retrieval, because it allows identification of the source of ocean backscatter that is not wind induced, e.g., sea ice, surface slicks, strong rain, current shear, and topography.

In total, 356 SAR imagettes where found were the differences in wind speed retrieved from SAR imagettes to the ECMWF analysis was $\geq 4 \mathrm{~ms}^{-1}$. Visual inspection of these SAR imagettes resulted in 19\% of the SAR imagettes affected by features not due to the wind field and most of these features attributed to ice or surface slicks. In Fig. 14, a subset of typical SAR imagettes is selected from the 356 SAR imagettes which depict features that are not due to the mean wind field. The SAR imagettes in the panel are ordered according to the inhomogeneity parameter. It can be seen that most of these are affected by ice and surface slicks. Additional image-analysis tools need to be developed to exclude these SAR imagettes from SAR wind retrieval.

\section{SUMMARY}

Since 1991, ERS SAR wave mode data have been acquired over the oceans on a global continuous basis. As the SAR wave mode data were only processed to SAR image spectra to measure ocean wave spectra, their full potential has been exploited only to a very limited extent. This study shows how SAR imagette data can be used to derive the ocean surface wind speed with and without a priori wind direction information. Therefore, two approaches are proposed, which are especially tailored to meet the needs of the ENVISAT ASAR wave mode. The first method is based on the well-known C-band SCAT model CMOD4 and is limited to C-band VV-polarized SAR systems. In case of the utilized ERS-2 SAR imagette data, a separate step for radiometric calibration had to be performed. The second approach is based on the application of NNs, which are used to parameterize the dependency of SAR intensity on the ocean surface wind and which are independent of radar frequency and po- larization. Both methods are strongly dependent on the relative stability of the SAR system.

The radiometric calibration of the SAR imagettes was performed by utilizing a new method that is based on the knowledge of the strong dependency of the NRCS on incidence angle and the ocean surface wind vector, which is given by the CMOD4. This method allows the accurate calibration of the spaceborne C-band SAR system on a short term basis (a few days of data). In contrast to conventional methods, all data acquired over the ocean surface can be considered for calibration and, therefore, enable continuous and global monitoring. Furthermore, this implies the opportunity to monitor short term calibration variations as well as orbital variations, which cannot be monitored with conventional methods.

Comparison of SAR-derived wind speeds to ERS-2 SCAT measurements utilizing the CMOD4 with a priori information on wind direction, resulted in a correlation of 0.95 and an rms error of $1.0 \mathrm{~ms}^{-1}$. If the wind direction is not considered the correlation is 0.87 with an rms error of $1.93 \mathrm{~ms}^{-1}$, whereby the error increases significantly with wind speeds. Comparisons applying an NN with a priori information on wind direction resulted in a correlation of 0.96 and an rms error of $0.93 \mathrm{~ms}^{-1}$ and a correlation of 0.87 and an rms error of $1.55 \mathrm{~ms}^{-1}$ if wind directions are not considered.

The main source of errors in wind speed retrieval is due to the lack of SAR imagettes available at high wind speeds for radiometric calibration and training of the NNs. Another source of error is due to SAR imagettes that passed the inhomogeneity filter and are effected by artifacts not due to the wind, e.g., sea ice and slicks.

For this study, only a very limited set of 34000 SAR imagettes was available. However, it is planned to reprocess the entire set of ten years of ERS SAR wave mode data and use them together with the very similar, soon to be available, ASAR wave mode data from ENVISAT to investigate effects of global as well as local wind field climate change.

\section{ACKNOWLEDGMENT}

The ERS-2 SAR image mode raw data were kindly provided by the European Space Agency.

\section{REFERENCES}

[1] S. Lehner, J. Horstmann, W. Koch, and W. Rosenthal, "Mesoscale wind measurements using recalibrated ERS SAR images," J. Geophys. Res., vol. 103, pp. 7847-7856, 1998.

[2] J. Horstmann, W. Koch, S. Lehner, and R. Tonboe, "Ocean winds from RADARSAT-1 scanSAR," Can. J. Remote Sens., vol. 28, no. 3, pp. 524-533, 2002.

[3] P. Heimbach, S. Hasselmann, and K. Hasselmann, "Statistical analysis and intercomparison with WAM model data of three years of global ERS-1 SAR wave mode spectral retrievals," J. Geophys. Res., vol. 103, pp. 7931-7977, 1998

[4] C. Mastenbroek and C. F. de Valk, "A semi-parametric algorithm to retrieve ocean wave spectra from synthetic aperture radar," J. Geophys. Res., pp. 3497-3516, 2000.

[5] J. Schuiz-Stellenfleth and S. Lehner, "Spaceborne synthetic aperture radar observations of ocean waves travelling into sea ice," J. Geophys. Res., vol. 107, pp. 10 1029-10 1039, 2002.

[6] A. Scoon, I. S. Robinson, and P. Meadows, "Demonstration of an improved calibration scheme for ERS-1 SAR imagery using a scatterometer wind model," Int. J. Remote Sens., vol. 17, pp. 413-418, 1995. 
[7] P. W. Vachon and F. Dobson, "Validation of wind vector retrieval from ERS-1 SAR images over the ocean," Global Atmos. Ocean Syst., vol. 5, pp. 177-187, 1996.

[8] J. Horstmann, W. Koch, S. Lehner, and R. Tonboe, "Wind retrieval over the ocean using synthetic aperture radar with C-band HH polarization," IEEE Trans. Geosci. Remote Sensing, vol. 38, pp. 2122-2131, Sept. 2000.

[9] F. Monaldo, D. Thompson, R. Beal, W. Pichel, and P. Clemente-Colon, "Comparison of SAR-derived wind speed with model predictions and ocean buoy measurements," IEEE Trans. Geosci. Remote Sensing, vol. 39 , pp. 2587-2600, Dec. 2002

[10] A. Stoffelen and D. Anderson, "Scatterometer data interpretation: Estimation and validation of the transfer function CMOD4," J. Geophys. Res., vol. 102, pp. 5767-5780, 1997.

[11] Y. Quilfen, B. Chapron, T. Elfouhaily, K. Katsaros, and J. Tournadre, "Observation of tropical cyclones by high-resolution scatterometry," $J$. Geophys. Res., vol. 103, pp. 7767-7786, 1998.

[12] J. Horstmann, S. Lehner, and H. Schiller, "Global wind speed retrieval from complex SAR data using scatterometer models and neural networks," in Proc. IGARSS, Sydney, Australia, 2001.

[13] H. Schiller and R. Doerffer, "Neural network for emulation of an inverse model-Operational derivation of case II water properties from MERIS data," Int. J. Remote Sens., vol. 20, no. 9, pp. 1735-1746, 1999.

[14] S. Thiria, F. Badran, C. Mejia, and M. Crepon, "A neural network approach for modeling non linear transfer functions: Application for wind retrieval from spaceborne scatterometer data," J. Geophys. Res., vol. 98, pp. 22 827-22 841, 1993.

[15] P. Richaume, F. Badran, M. Crepon, C. Mejia, H. Roquet, and S. Thiria, "Neural network wind retrieval from ERS-1 scatterometer data," J. Geophys. Res., vol. 105, pp. 8737-8751, 2000.

[16] V. Kerbaol, B. Chapron, and P. W. Vachon, "Analysis of ERS-1/2 synthetic aperture radar wave mode imagettes," J. Geophys. Res., vol. 103, pp. 7833-7846, 1998

[17] S. Lehner, J. Schulz-Stellenfleth, B. Schättler, H. Breit, and J. Horstmann, "Wind and wave measurements using complex ERS-2 SAR wave mode data," IEEE Trans. Geosci. Remote Sensing, vol. 38, pp. 2246-2257, Sept. 2000.

[18] H. Essen, "Theoretical investigation on the impact of long surface waves on empirical ERS-1/2 scatterometer models," Int. J. Remote Sens., vol. 21, pp. 1633-1656, 2000

[19] H. Breit, B. Schättler, and U. Steinbrecher, "A high precision workstation-based chirp scaling SAR processor," in Proc. IGARSS, Singapore, 1997.

[20] A. Bentamy, Y. Quilfen, and P. Queuffeulou, "Calibration of the ERS-1 Scatterometer C-Band Model," Institute Française de Recherche pour l'Exploitation de la Mer, France, IFREMER DRO/OS-94-01, 1994.

[21] S. Lehner, J. Schulz-Stellenfleth, A. Niedermeier, J. Horstmann, and W. Rosenthal, "Extreme waves observed by synthetic aperture radar," in Proc. 4th Int. Symp. Ocean Wave Measurement and Analysis, vol. 1, San Francisco, CA, 2001, pp. 125-134.

[22] J. Honerkamp, Stochastic Dynamical Systems. Berlin, Germany: VCH Verlagsge-sellschaft mbH, 1993

[23] W. Donnelly, J. Carswell, R. McIntosh, P. Chang, and J. Wilkerson, "Revised ocean backscatter models at $\mathrm{C}$ and $\mathrm{Ku}$ band under high wind conditions," J. Geophys. Res., vol. 104, pp. 11 485-11 498, 1999.

[24] P. Meadows, H. Laur, J. Sanchez, and B. Schattler, "The ERS SAR performances," in Proc. CEOS SAR Calibration and Validation Workshop '98. Noordwijk, The Netherlands, 1998, pp. 223-232.

[25] P. Meadows and P. Wright, "ERS-1 SAR analogue to digital convertor saturation," in Proc. CEOS SAR Calibration and Validation Workshop '94, 1994, pp. 24-37.

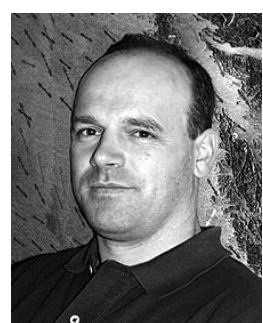

Jochen Horstmann received the Diploma degree in physical oceanography and the $\mathrm{Ph} . \mathrm{D}$. degree in earth sciences, both from the University of Hamburg, Hamburg, Germany, in 1997 and 2002, respectively.

He is currently a Research Scientist with the Institute for Coastal Research, GKSS Research Center, Geesthacht, Germany. In 1995, he joined the Coupled Model System group, GKSS. In 2002, he was a Visiting Scientist with the Applied Physics Lab, Johns Hopkins University, Baltimore, MD, and the National Environmental Satellite, Data, and Information Service, National Oceanic and Atmospheric Administration, Washington, DC. His main research interests are in extraction of geophysical parameters from RAR, $\mathrm{SAR}$, and interferometric SAR.

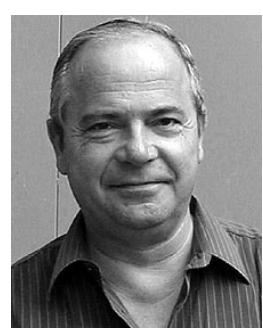

Helmut Schiller received the Diploma degree in physics and the Ph.D. degree in experimental high-energy physics, in 1966 and 1970, respectively, both from the Humboldt University of Berlin, Berlin, Germany.

He was Research Scientist at the Academy of Sciences of the former GDR developing data acquisition and evaluation methods for high-energy physics experiments. His research interests are methods for high-dimensional data analysis. He joined the GKSS Research Center, Geesthacht, Germany, in 1989, where he is now a Research Scientist in the bio-optical remote sensing group, working on the development of algorithms for the retrieval of concentrations of water constituents from satellite data.

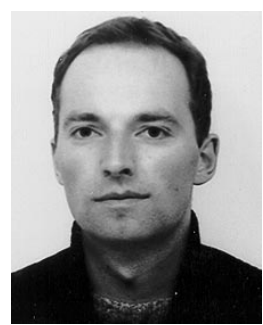

Johannes Schulz-Stellenfleth received the Diploma degree in applied mathematics from the University of Hamburg, Hamburg, Germany, in 1996.

He joined the German Aerospace Center (DLR), Oberpfaffenhofen, Germany, in late 1996. He is currently a Research Scientist with the Remote Sensing Technology Institute (IMF) at DLR. The main interest of his present work is the use of complex SAR data to derive two-dimensional ocean wave spectra. Apart from that, he is working on the application of cross track interferometric (InSAR) data to measure sea surface elevation models.

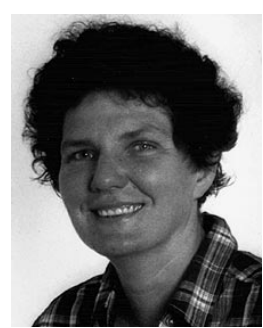

Susanne Lehner (M'01) received the M.S. degree in applied mathematics from Brunel University, Uxbridge, U.K., in 1979, and the Ph.D. degree in geophysics from the University of Hamburg, Hamburg, Germany, in 1984.

She was a Research Scientist with the Max-Planck Institute for Climatology, Hamburg, Germany, and in 1996, joined the German Aerospace Center (DLR/DFD), Oberpfaffenhofen, Germany. She is currently a Research Scientist in marine remote sensing at the Remote Sensing Technology Institute (DLR/IFM), working on the development of algorithms determining marine parameters from SAR 